\title{
Transabdominal amnioinfusion in the correction of oligohydramnios following twin-to-singleton reduction - A report of two cases
}

DOI 10.1515/crpm-2016-0021

Received February 2, 2016. Accepted October 14, 2016. Previously published online November 29, 2016.

\section{Abstract}

Background: On rare occasions, elective iatrogenic reduction of a dichorionic twin is performed due to maternal request and in order to improve the perinatal outcome.

Materials and methods: Nine twin-to-singleton reductions were identified retrospectively at the Feto-maternal Unit, University of Szeged, Hungary, between December 1997 and June 2015. A post-procedure, routine weekly sonographic scan revealed severe oligohydramnios in two out of the nine cases $(22.2 \%)$ and amnioinfusion was performed in the mid-trimester to prolong gestation. The fetus survived in one case (11.1\%) and the pregnancy continued until preterm birth.

Conclusion: A repeated sonographic follow-up for an early diagnosis of oligohydramnios is feasible to avoid miscarriage after artificial embryo reduction.

Keywords: Amnioinfusion; embryo reduction; oligohydramnios; serial sonographic follow-up.

\section{Introduction}

An escalating prevalence of infertility results in more infants being conceived with assisted reproductive technologies, leading to a dramatic increase in multifetal gestations [1]. Multiple births frequently have adverse perinatal sequelae, but the iatrogenic termination of

János Sikovanyecz, Attila Keresztúri and Gábor Németh: These authors contributed equally to this work.

*Corresponding author: Zoltan Kozinszky, MD, PhD, Kvinnokliniken, Landstinget Blekinge, Lasarettvägen, 37181 Karlskrona, Sweden; and Department of Obstetrics and Gynecology, Blekinge Hospital, Karlskrona, Sweden, Tel.: +46730720835,

E-mail:kozinszky@gmail.com

Norbert Pásztor, Virág Márton, János Sikovanyecz, Attila Keresztúri and Gábor Németh: Department of Obstetrics and Gynecology,

Faculty of General Medicine, University of Szeged, Szeged, Hungary one or more presumably healthy fetuses is advocated for avoiding an array of adverse maternal and fetal outcomes [2].

Some evidence suggests that reduced dichorionic twins are at lower risk compared to that of their unreduced counterparts [3, 4]. Hence, electively performed iatrogenic twin-to-singleton reduction is employed as a treatment option to prolong gestation, particularly for those mothers who do not want more than one infant or have serious medical/psychiatric illness [3]. The pregnancy loss rate of this intervention is well documented, but there is a paucity of information on post-procedure oligohydramnios following early/late fetal reduction from twins to singletons [3-5]. The purpose of this study was to assess the clinical value of detecting oligohydramnios following artificial embryo reduction of twin pregnancies and the treatment of transabdominal fluid replacement (amnioinfusion).

\section{Materials and methods}

In our retrospective case series, we have reviewed nine elective twin-to-singleton reductions performed in the first trimester at the Feto-maternal Unit of the Department of Obstetrics and Gynecology, University of Szeged, Hungary, between December 1997 and June 2015. Hungarian regulations allow twin reduction for mothers who do not want more than one infant. Our work was carried out in accordance with the Code of Ethics of the World Medical Association (Declaration of Helsinki) for experiments involving humans, and our study has been approved by the institutional research Ethics Committee. Informed consent was obtained from all participants.

The study group included dizygotic twin pregnancies who underwent elective reductions, in an attempt to improve the expected poor outcome of their multiple pregnancies. The fetus chosen for in utero intervention would be the one with suspicious ultrasonographic (US) findings, such as enlarged ( $\geq 3 \mathrm{~mm}$ ) nuchal translucency or delayed growth in comparison with its co-twin. In the absence of any pathognomic signs of abnormalities (Kretz Sonoace 8000 , Voluson 730 Pro, Zipf, Austria), the more readily accessible fetus was chosen usually the one in a ventral and fundal location. Amniotic fluid volume (AFV) was appropriate for both fetuses and there was no sign of hydrops. No symptoms indicative of incomplete miscarriage were present and no clear signs of maternal or fetal infection (maternal tachycardia $>100 / \mathrm{min}$, maternal temperature $>38^{\circ} \mathrm{C}$, 
maternal white blood cell count $>15,000 / \mathrm{mL}$, maternal C-reactive protein $>20 \mathrm{mg} / \mathrm{L}$, uterine tenderness, foul-smelling vaginal discharge, fetal tachycardia $>160 \mathrm{bpm}$ ) were noted.

Terminating one of the fetuses was achieved by intrathoracic injection of $\mathrm{KCl} 10 \%(0.6-3.1 \mathrm{~mL})$ via a Chiba $20^{*} 150-\mathrm{mm}$ needle (Neomed Corporations, Debrecen, Hungary), which took place under continuous US control between weeks 11 and 12 of gestation via a transabdominal approach, after measuring nuchal translucency. Thereafter, the patients received a weekly US scan until the viability of the remaining fetus ( $22^{\text {nd }}$ week of gestation), and then every second week until the $36^{\text {th }}$ week. US was performed in order to assess the growth and well-being of the fetus, as well as to identify oligo hydramnios during mid-to-late gestation that could require further intervention. The diagnosis of severe pathologic deficiency of the amniotic fluid (AF) was based on the AF index (AFI). AFI values lower than $5 \mathrm{~cm}$ necessitated performing transabdominal amnioinfusion (TAAI) [6]. Persistent severe oligohydramnios prior to 22-24 weeks may impede fetal lung development, predispose to limb contractures and prognosticate extreme prematurity [7]. A certain volume of $37^{\circ} \mathrm{C}$ saline solution $(0.9 \% \mathrm{NaCl})$ was infused transabdominally into the amniotic cavity under continuous US control in order to restore the AFI to $>8 \mathrm{~cm}$, with the aim of prolonging gestation in all cases of oligohydramnios. Eligibility criteria for TAAI were the same as for those for fetal reduction, with the addition of no clinical sign of occult/ apparent preterm premature rupture of membranes and no suspicion of placental abruption (uterine tenderness/contractions and bleed ing episodes) [6]. The intervention procedure was described in our previous article [7]. In brief, under sterile circumstances, a needle was introduced transabdominally into the amniotic cavity and a variable volume of $37^{\circ} \mathrm{C}$ saline solution was infused into the uterus under continuous US control.

\section{Results}

Out of the nine elective reductions performed, two pregnancies $(22.2 \%)$ were complicated, with mid-trimester oligohydramnios for the remaining healthy fetuses. The overall pregnancy loss rate was $11.1 \%$. A healthy 35 -yearold nulliparous woman with a dichorionic diamniotic pregnancy requested fetal reduction at the $12^{\text {th }}$ week of gestation, which was carried out without any complications. Following the initial intervention, the patient received repeated US scans that showed severe oligohydramnios at $18+5$ weeks of gestation. Normalization of the AF level was performed by instilling $180 \mathrm{~mL}$ saline solution via TAAI under real-time guidance, and it resulted in retroamnial filling. The pregnancy finished with miscarriage in the second trimester as a consequence of rupture of membranes within one day and clinical signs of chorioamnionitis that required a surgical uterine evacuation. The patient was administered intravenous antibiotics managed at ward level and the combined treatment was without complications. A placenta swab later indicated intrauterine mycoplasma infection. Fetopsy and placental histological examination did not reveal any pathological malformation of the remaining fetus.

A 32-year-old non-obese woman with no pre-existing medical condition was referred to our unit at $11+3$ weeks of gestation with a clinically unremarkable dichorionic, diamniotic twin pregnancy for a detailed anatomy scan and reduction to a singleton. The patient had had a previous full-term cesarean section. Both the current and previous pregnancies were achieved by in vitro fertilization. Multifetal pregnancy reduction had been done according to the standard guidelines without any complications. In the index pregnancy, the $18^{\text {th }}$ week anomaly scan was reported as normal, the glucose tolerance test was negative and the mother had been at low risk on combined first trimester screening for Down's syndrome. The pregnancy was uncomplicated until $24+5$ weeks of gestation when severe oligohydramnios was detected at the serial sonographic follow-up accompanied by no other contributing maternal condition (i.e. symptoms of intrauterine infection, hypertension or other complications) or fetal malformation (i.e. malformations or growth retardation). Prior to the procedure, AFI was $2 \mathrm{~cm}$, and $500 \mathrm{~mL}$ saline solution was infused until a normal amount of fluid was restored, exceeding the upper limit of moderate oligohydramnios $(\mathrm{AFI}>8 \mathrm{~cm}$ ). No transplacental puncture was needed as the posterior placenta was described and the patient denied any drug abuse. Serology for cytomegalovirus, toxoplasmosis, syphilis, rubella and parvovirus B19, thyroid function tests, thrombophilia and autoantibody screens during pregnancy were also negative. Repeated measurements of the AF demonstrated that oligohydramnios did not recur. Doppler velocimetric indices of the uterine, umbilical, renal and middle cerebral arteries were within normal ranges in the second trimester (https:// fetalmedicine.org/var/uploads/Doppler-in-Obstetrics. pdf). The patient was admitted to the maternity ward due to a subsequent rupture of membranes $72 \mathrm{~h}$ after TAAI. As per the policy of expectant management, to try to gain additional time for the fetus in utero, the patient obtained prophylactic antibiotic treatment. Chorioamnionitis had not emerged in the clinical course and TAAI had not been repeated as US scans confirmed only moderate oligohydramnios $(\mathrm{AFI}=5-8 \mathrm{~cm})$. The pregnancy continued until premature delivery at $29+0$ weeks of gestation when the patient had lower abdominal pain and several antecedent episodes of small vaginal bleeding. The patient exhibited painful uterine tenderness during physical examination and abdominal US demonstrated a $2.4 \mathrm{~cm} \times 2.8 \mathrm{~cm}$ hematoma behind the posteriorly attached placenta. Minimal bleeding through the cervical os was recorded during pelvic examination. The mother delivered a healthy male 
neonate with a birthweight of 1380 grams via an immediately performed cesarean section. The infant had no "oligohydramnios sequence" or pathologies caused by prolonged exposure to oligohydramnios and is currently a healthy 8-year-old. The postpartum period was uneventful. The mother did not smoke and had no history of abruption or thromboembolism. Microbiological examination of high vaginal, placental and mid-stream urinalysis revealed no infection. Gross examination of the placenta revealed blood clots tenuously adhered to the placental floor and old fibrin and necrotic tissue on the decidual plate with infarcted areas as in acute abruption. Microscopic analysis demonstrated hemorrhage and edema in the decidua and villous stroma and intervillous thrombi [8].

\section{Discussion}

To our knowledge, this is the first report of oligohydramnios as a sporadic post-procedural clinical finding after an artificial fetal reduction in a twin pregnancy. The rate of oligohydramnios was $22 \%$ in our dataset; however, a relatively low number of participants were included in our study. One of our cases was a consequence of intrauterine infection. However, the exact pathomechanism of the AF anomaly remained uncertain, and it can be considered unexplained or intervention-related in the other case. Another possible explanation for decreased fluid provision for the remaining fetus could be the altered uteroplacental circulation, but we could not notice any pathology in the 2-D Doppler-indices (https://fetalmedicine.org/var/ uploads/Doppler-in-Obstetrics.pdf).

The etiology of placental abruption also remains speculative in our case; however, a history of a cesarean delivery and the two previous invasive procedures represent a clear predisposition [9]. In addition, oligohydramnios is a predisposing factor for placental abruption, and vice versa, small placental hematomas pose a risk of oligohydramnios [8]. It is pivotal to detect the oligohydramnios in time because this obstetric condition could be corrected via amnioinfusion in order to prevent uterine contractions and a subsequent pregnancy loss [7].

Some evidence suggests that reduced dichorionic twins are at lower risk compared to their unreduced counterparts $[3,4]$. The risk of loss of the remaining fetus associated with the iatrogenic twin-to-singleton reduction due to the post-procedural amniotic leakage and chorioamnionitis is a major concern [2]. Early elective reduction of twins is associated with a less-than-10\% pregnancy loss, and procedure-to-loss interval is often 4-8 weeks [5], whereas others reported a miscarriage rate of $2.5 \%-11.9 \%$ following fetal reduction [3, 4].

Although severe oligohydramnios of the surviving fetus after elective reduction is not investigated [3-5], midtrimester oligohydramnios poses a risk of perinatal mortality as high as $80 \%$ [7]. It is noteworthy that amnioinfusion can significantly prolong the pregnancy and artificially increased AFV could prevent $49.1 \%$ of the fetal deaths [7].

Our data highlight that a series of weekly repeated sonographic follow-ups are beneficial in order to detect non-infectious oligohydramnios that could be related to mid-trimester post-procedure miscarriage. Further research is needed to determine the post-procedural incidence of this preventable, unfavorable antenatal condition and to establish the benefit of amnioinfusion in the improvement of neonatal survival.

\section{Author's statement}

Conflict of interest: Authors state no conflict of interest.

\section{Material and methods}

Informed consent: Informed consent has been obtained from all individuals included in this study.

Ethical approval: The research related to human use has been done in compliance with all the relevant national regulations, institutional policies and in accordance the tenets of the Helsinki Declaration, and has been approved by the authors' institutional review board or equivalent committee.

\section{References}

[1] Sunderam S, Kissin DM, Crawford SB, Folger SG, Jamieson DJ, Barfield WD, et al. Assisted reproductive technology surveillance - United States, 2011. MMWR Surveill Summ 2014;63:1-28.

[2] Evans MI, Berkowitz R, Wapner R, Carpenter RJ, Goldberg JD, Ayoub MA, et al. Multifetal pregnancy reduction (MFPR): Improved outcomes with increased experience. Am J Obstet Gynecol 2001;184:97-103.

[3] van de Mheen L, Everwijn SM, Knapen MF, Haak MC, Engels MA, Manten GT, et al. Pregnancy outcome after fetal reduction in women with a dichorionic twin pregnancy. Hum Reprod 2015;30:1807-12.

[4] Evans MI, Kaufman M, Urban AJ, Britt DW, Fletcher JC. Fetal reduction from twins to a singleton: a reasonable consideration. Obstet Gynecol 2004;104:102-9.

[5] Yaron Y, Johnson KD, Bryant-Greenwood PK, Kramer RL, Johnson MP, Evans MI. Selective termination and elective reduction in twin pregnancies: 10 years experience at a single centre. Hum Reprod 1998;13:2301-4.

[6] Kozinszky Z, Pásztor N, Vanya M, Sikovanyecz J, Pál A. Management of severe idiopathic oligohydramnios: is antepartum transabdominal amnioinfusion really a treatment option? ) Matern Fetal Neonatal Med 2013;26:383-7. 
[7] Kozinszky Z, Sikovanyecz J, Pásztor N. Severe midtrimester oligohydramnios: treatment strategies. Curr Opin Obstet Gynecol 2014;26:67-76.

[8] Elsasser DA, Ananth CV, Prasad V, Vintzileos AM. New Jersey. Placental Abruption Study Investigators. Diagnosis of placental abruption: relationship between clinical and hystopathological findings. Eur J Obstet Gynecol Reprod Biol 2010;148:125-30.

[9] Minna T, Mika G, Tiina L, Marjo M, Sture A, Olavi Y, et al. Risk for placental abruption following amniocentesis and chorionic villus sampling. Prenat Diagn 2011;31:410-2. 